Publisher: Taylor \& Francis

Journal: International Journal of Control

DOI: $10.1080 / 00207179.2019 .1605203$

\title{
Enclosing a moving target with an optimally rotated and scaled multiagent pattern
}

\author{
Miguel Aranda ${ }^{\mathrm{a}}$, Youcef Mezouara ${ }^{\mathrm{a}}$, Gonzalo López-Nicolás ${ }^{\mathrm{b}}$ and Carlos Sagüés ${ }^{\mathrm{b}}$ \\ ${ }^{a}$ Université Clermont Auvergne, CNRS, SIGMA Clermont, Institut Pascal, F-63000 \\ Clermont-Ferrand, France (e-mail: firstname.lastname@ sigma-clermont.fr); ${ }^{b}$ Instituto de \\ Investigación en Ingeniería de Aragón - Universidad de Zaragoza, C/ María de Luna 1, E-50018
}

Zaragoza, Spain (e-mail: gonlopez@ unizar.es, csagues@unizar.es).

Received 22 Nov 2017, Revised 17 Dec 2018, Accepted 01 Apr 2019

\author{
ARTICLE HISTORY \\ Compiled April 8, 2019
}

\begin{abstract}
We propose a novel control method to enclose a moving target in a two-dimensional setting with a team of agents forming a prescribed geometric pattern. The approach optimises a measure of the overall agent motion costs, via the minimisation of a suitably defined cost function encapsulating the pattern rotation and scaling. We propose two control laws which use global information and make the agents exponentially converge to the prescribed formation with an optimal scale that remains constant, while the team's centroid tracks the target. One control law results in a multiagent pattern that keeps a constant orientation in the workspace; for the other, the pattern rotates with constant speed. These behaviors, whose optimality and steadiness are very relevant for the task addressed, occur independently from the target's velocity. Moreover, the methodology does not require distance measurements, common coordinate references, or communications. We also present formal guarantees of collision avoidance for the proposed approach. Illustrative simulation examples are provided.
\end{abstract}

\section{KEYWORDS}

Multiagent systems, Target enclosing, Target tracking, Formation stabilisation

\section{Introduction}

This paper addresses the problem of enclosing a moving physical entity using multiple mobile agents, which allows to acquire and maintain rich perception of this target or to escort it. There has been notable recent research interest in achieving target-tracking behaviors with multirobot systems (Hausman, Müller, Hariharan, Ayanian, and Sukhatme (2015); Khan, Rinner, and Cavallaro (2017); Robin and Lacroix (2016)), due to the important applications they enable in the context of, e.g., autonomous monitoring or surveillance tasks. Next, we highlight the novel aspects of our proposed approach and situate it in the context of the relevant related work in this area.

This work was supported by the French Government via programs FUI (project Aerostrip) and Investissements d'Avenir (I-SITE project CAP 20-25 - MaRoC), and by the Spanish Government/European Union through project DPI2015-69376-R. 


\subsection{Statement of contribution}

We propose two control laws to enclose a moving target with a team of single-integrator kinematic agents in two-dimensional space. For both laws, the agents achieve exponential convergence to a formation pattern with optimal orientation and scale. More precisely, these parameters are optimal in the sense that they minimise at any time the sum of squared distances that have to be traveled to reach the desired formation pattern. A key aspect of our contribution is that this optimality is obtained without needing common reference frames, communications, or distance measurements. Our analysis shows that - under arbitrary target motions- one control law leads to a stable formation pattern, while the other results in the pattern gyrating at constant speed. We also provide guarantees on the avoidance of collisions. Under the two proposed control laws, the scale of the pattern remains constant and the centroid of the group tracks the target's position.

These behaviors are very interesting in various respects, discussed next. As the scale and orientation of the pattern are optimal, the enclosing of the target is achieved efficiently. The steadiness of the behavior in terms of the relative geometry of the elements (constant or uniformly varying pattern orientation, and constant scale) ensures that stable sensing and communications will be maintained among agents. Due to this uniform behavior, our system may be thought of as a mobile implementation of the sensing cage used in optical motion capture systems (Guerra-Filho (2005)), where the static relative geometry of the sensors allows highly precise and occlusion-free reconstruction of all parts of a target. Another advantage concerns safety and comfort, which are of singular importance, e.g., if the enclosed target is a human. The human's perceived comfort when navigating accompanied by robots is increased when these robots exhibit uniform and predictable behaviors with small relative accelerations (Kruse, Pandey, Alami, and Kirsch (2013)). Thanks to its steadiness and the decoupling of the interagent motions from the movements of the human target, our method provides these characteristics.

We choose to employ global information in our controller. In particular, every agent needs to measure the relative position of all the other agents and of the target. Using global information for the problem we address is interesting because, by exploiting it in an optimal manner, we obtain the key advantages in performance detailed in the paragraph above. Moreover, it is a reasonable choice given the conditions of the scenario we consider: tracking a single target generally involves few agents (i.e., scalability is not an issue), and the controller we present is decentralised. By this latter term we mean, as is common in the literature of multiagent systems, that there is no central unit in the system (i.e., no single point of failure) and there is no need for communications between the entities that compute the controller (i.e., the agents). This is the case for us because each agent can obtain the information it needs operating completely autonomously: it can simply use onboard sensors and its own independent measurement frame, and thus does not have to rely in any way on other agents or external systems to implement the proposed method. In addition, ours is a computationally simple control method. These modest requirements increase the applicability of the approach by robots endowed with reasonable sensing and computation resources.

\subsection{Related work}

We review in this section works in the literature that relate in different and substantial ways to the approach we propose. Several existing methods allow to achieve target circumnavigation by a multiagent team with constrained geometry (generally, circular). Some of them consider the velocity of the target to be zero (Franchi, Stegagno, Rocco, and Oriolo (2010); Marasco, Givigi, and Rabbath (2012); Montijano, Priolo, Gasparri, and Sagüés (2013)), or 
constrained or known (Franchi, Stegagno, and Oriolo (2016); Guo, Yan, and Lin (2010); Mas, Li, Acain, and Kitts (2009); Shames, Fidan, and Anderson (2011); Shi, Li, and Teo (2015); Swartling, Shames, Johansson, and Dimarogonas (2014)), and in some cases only bearing sensors are needed by the agents (Mallik, Daingade, and Sinha (2016); Zheng, Liu, and Sun (2015)). In contrast, the method we propose does not constrain the prescribed team geometry. Having this freedom in a multirobot system is very relevant as it can be used to ensure suitable interactions between its elements. One can, e.g., add more agents to a team while preserving safety distances, or consider agents with heterogeneous sensory capabilities -i.e., different optimal distances to the target in terms of sensing quality-. Noncircular formations require more sophisticated coordination mechanisms such as the one naturally provided by our method. We also alleviate here the constraints on the target's motion and decouple it from the dynamics of the group of agents, thus allowing steady formation behaviors. Other work similarly addresses target observation in 3D space, with further constraints on target motions and team behaviors (Aranda, López-Nicolás, Sagüés, and Zavlanos (2014); Poiesi and Cavallaro (2015)).

To allow the target-tracking team of agents to attain an arbitrarily defined geometric pattern, the method we propose exploits gradient-based formation control (Oh, Park, and Ahn (2015)). Each agent can compute locally its motion commands. Therefore, it is possible to run this method relying solely on simple onboard sensors (e.g., vision). We avoid the need for the agents to continuously maintain virtual agreed common team references, which are subject to measurement errors and/or require communications. Various existing formation control approaches similarly allow the agents to steer their relative distances or angles without requiring common references (Anderson, Yu, Fidan, and Hendrickx (2008); Eren (2012); Garcia de Marina, Jayawardhana, and Cao (2016); Krick, Broucke, and Francis (2009); Lin, Wang, Han, and Fu (2014); Tian and Wang (2013); Zelazo, Giordano, and Franchi (2015)). These controllers were designed for distributed scenarios (which provides benefits in robustness and scalability) and therefore, they are not the best option to choose when global information is available. That is to say, what they optimise is a combination of partial cost functions that typically encompass a pair of agents and do not consider an overall optimal motion goal.

To get an intuitive idea, consider a team of three agents $a, b$ and $c$. A motion strategy that uses separately information of pairs $a-b, a-c, b-c$ (as with a typical distributed system) cannot generate globally optimal motions, because each separate part lacks information about the state of one of the agents. In contrast, optimal motions can be obtained by using a cost function that considers jointly the states of the three agents. Our controller provides, and suitably exploits, such optimality. Note that in our discussion we are referring to distributed motion control (typically, gradient-based), and not to distributed optimisation algorithms. Such algorithms can find optimal solutions to problems like the one we address, but running them for online control tasks requires inter-agent communications and considerable computational effort. We do not use optimisation algorithms and our controller does not have these requirements. We remark as well that its computational simplicity and analytical properties make our method interesting when compared with alternative methodologies that can also be appropriate to solve the problem such as Model Predictive Control.

Containment control is a related problem that has also received much attention (Cao, Stuart, Ren, and Meng (2011); Ji, Ferrari-Trecate, Egerstedt, and Buffa (2008); Wang, Liu, Xiao, and Lin (2017); Wang, Liu, Xiao, and Shen (2018)). The target enclosing problem considered here differs in important respects. In containment control, one considers leaders (enclosing agents) and followers (enclosed agents) and designs control strategies for both types. In contrast, for us the target to be enclosed is an uncontrolled, external agent. Moreover, the containment goal is defined as keeping the followers in the convex hull of the 
leaders; whereas in target enclosing one defines a specific desired location of the target (typically, the centroid of the multiagent team). The problem we address is also connected with that of achieving consensus with a variable reference, studied in, e.g., (Cao, Zhang, Li, and Chen (2017); Ren (2010)).

The global cost function we define encodes the sum of squared distances the agents have to travel to form the desired pattern, which is chosen with optimal orientation and scale via solving a Procrustes shape-alignment problem (Gower and Dijksterhuis (2004)). Similar optimisations -without studying target tracking and variable/unknown scales as we do here- have been used to control a formation (Aranda et al. (2014) Aranda, López-Nicolás, Sagüés, and Zavlanos (2015); Macdonald (2011)) or estimate its error (Ze-Su, Jie, and Jian (2012)). In other related work, several authors have studied how to optimise a formation's geometry to maximise the quality of the collective perception of a target, typically using the estimation covariance. Works in this area have considered different bearing or range sensors (Bishop, Fidan, Anderson, Doanay, and Pathirana (2010); Zhao, Chen, and Lee (2013)) and studied how to define appropriate agent motion policies to achieve the optimal formation (Martínez and Bullo (2006); Zhou and Roumeliotis (2011)). As explained above, the formation optimisation considered here is of a different nature, as it concerns the agents' motion costs.

The paper is organised as follows. Section 2 states the addressed problem. In Section 3 we describe the computation of the optimal pattern, while Section 4 introduces the proposed control laws. These are analysed in Section 5, where the results on formation achievement and target tracking are given. Section 6 describes the implementation details of our approach, whose behavior is illustrated in simulation in Section 7. Section 8 concludes the paper.

\section{Problem statement}

We consider a two-dimensional Euclidean space with a fixed global reference frame where all quantities will be expressed. The proposed system consists of $N-1$ mobile agents, identified by indexes $i \in\{1, \ldots, N-1\}$ and obeying single-integrator kinematics, i.e.:

$$
\dot{\mathbf{q}}_{\mathbf{i}}=\mathbf{u}_{\mathbf{i}}
$$

(boldface font is used for multidimensional variables), where $\mathbf{q}_{\mathbf{i}} \in \mathbb{R}^{2}$ denotes agent $i^{\prime} s$ position vector and $\mathbf{u}_{\mathbf{i}} \in \mathbb{R}^{2}$ is its control input. Let us denote as $\mathbf{p}_{\mathbf{q}} \in \mathbb{R}^{2}$ the centroid of the agent positions. The target is defined as a point element that the agents must enclose, with position denoted as $\mathbf{q}_{\mathbf{N}} \in \mathbb{R}^{2}$. The target moves with arbitrary finite-norm velocity:

$$
\dot{\mathbf{q}}_{\mathbf{N}}=\mathbf{v}_{\mathbf{t}} \text {. }
$$

We define $N /$ as the set of $N-1$ agents, and $\mathscr{N}$ as the set that includes all agents and the target. We specify a desired geometric pattern for the agents in their configuration space via relative position vectors: let us denote as $\mathbf{c}_{\mathbf{j i}}, \in \mathbb{R}^{2} \forall i, j \in \mathscr{N}_{-}$, the vector from $i$ to $j$ in this prescribed pattern. These vectors are assumed to be nonzero for $i \neq j$. Then, the agents are forming the pattern if and only if there exist $\mathbf{R}_{\mathbf{p}} \in S O(2)$ and $s_{p}>0$ such that:

$$
\mathbf{q}_{\mathbf{i j}}=s_{p} \mathbf{R}_{\mathbf{p}} \mathbf{c}_{\mathbf{i j}}, \quad \forall i, j \in \mathscr{N}_{-},
$$

where we define $\mathbf{q}_{\mathbf{i j}}=\mathbf{q}_{\mathbf{i}}-\mathbf{q}_{\mathbf{j}}$. This can be interpreted as the current pattern of agent positions being equal to the desired pattern up to a similarity transformation. We also define desired vectors from the target to each of the $N-1$ agents: $\mathbf{c}_{\mathbf{i N}} \forall i \in \mathscr{N}_{-}$. The desired location of the 
target is the centroid of the agents, i.e., $\sum_{i \in \mathscr{N}_{-}} \mathbf{c}_{\mathbf{i N}}=\mathbf{0}$. This fact ensures that pattern rotations and scalings do not move the target from the team's centroid, and it also will be useful in our formal developments. The problem addressed is finding a motion strategy that drives the agents to the desired pattern while enclosing the moving target.

\section{Proposed optimal target enclosing strategy}

A way to address the design of a controller to achieve the objective expressed in (3) is by defining the following general cost function:

$$
\gamma_{g}=\frac{1}{2} \sum_{i \in \mathscr{N}} \sum_{j \in \mathscr{N}}\left\|\mathbf{q}_{\mathbf{i j}}-s_{g} \mathbf{R}_{\mathbf{g}}\left(\alpha_{g}\right) \mathbf{c}_{\mathbf{i j}}\right\|^{2},
$$

with $s_{g}$ being a positive scalar, $\alpha_{g} \in(-\pi, \pi]$ an angle, and $\mathbf{R}_{\mathbf{g}}=\left[\left(\cos \alpha_{g}\right) \sin \alpha_{g}\right)^{T}$ $\left.\left(-\sin \alpha_{g}, \cos \alpha_{g}\right)^{T}\right]$ a rotation matrix in the Special Orthogonal group of dimension two $S O(2)$. We note that the norm considered in this paper is the Euclidean one. This cost function is a sum of squared distances that expresses how separated the agents are from a configuration that represents a rotated and scaled version of the prescribed pattern, with the target at its centroid. We can now precisely specify our control goal, as follows.

Definition 3.1. The target enclosing task consists in minimising the cost function $\gamma_{g}$.

Let us explain the behavior encapsulated by our definition: when $\gamma_{g}$ is minimised, the target, along its motion, will be surrounded by the agents (since it lies exactly at their centroid when $\gamma_{g}=0$ ), and meanwhile the agents' relative positions will form a pattern having the shape we have prescribed. Clearly, a control strategy that descends along the defined cost function can make the agents reach the desired objective. The question is then how to suitably define $s_{g}$ and $\mathbf{R}_{\mathbf{g}}\left(\alpha_{g}\right)$. To optimise control efficiency, we propose to choose these parameters, at each time instant, as those for which the rotated and scaled pattern is the closest -in terms of $\gamma_{g}$ - to the set of current positions of the agents. Thus, we find the optimal rotation, $\mathbf{R}_{\mathbf{o}}=$ $\left[\left(\cos \alpha_{o}, \sin \alpha_{o}\right)^{T}\left(-\sin \alpha_{o}, \cos \alpha_{o}\right)^{T}\right]$, and scaling, $s_{o}$, of the pattern by solving the following optimisation problem:

$$
\left(\mathbf{R}_{\mathbf{0}}\left(\alpha_{o}\right), s_{o}\right)=\underset{\mathbf{R}_{\mathbf{g}}\left(\alpha_{g}\left(\mathbf{q}_{\mathbf{i j}}\right)\right) \in S O(2), s_{g}\left(\mathbf{q}_{\mathbf{i j}}\right)>0}{\arg \min } \gamma_{g}\left(\mathbf{R}_{\mathbf{g}}\left(\alpha_{g}\left(\mathbf{q}_{\mathbf{i j}}\right)\right), s_{g}\left(\mathbf{q}_{\mathbf{i j}}\right), \mathbf{q}_{\mathbf{i j}}\right),
$$

which is computed at every time instant $t$ for the current positions $\mathbf{q}_{\mathbf{i j}}(t)$ of the agents and target (i.e., $\forall i, j \in \mathscr{N}$ ). $\gamma_{g}$ is actually equivalent to the function that is optimised in the orthogonal Procrustes problem (Gower and Dijksterhuis (2004)), and the stated optimisation problem has an analytical solution for the pair $\left(\mathbf{R}_{\mathbf{o}}\left(\alpha_{o}\right), s_{o}\right)$ that is unique except for degenerate cases that will be ruled out in the analysis we provide. For clarity and completeness, we describe next the computation of the solution in terms of the formulation used in this paper. To calculate the rotation angle $\alpha_{o}$ that minimises $\gamma_{g}$, we will solve $\frac{\partial \gamma_{g}}{\partial \alpha_{g}}=0$. Using that $\frac{d \mathbf{R}_{\mathbf{g}}\left(\alpha_{g}\right)}{d \alpha_{g}}=\mathbf{A} \mathbf{R}_{\mathbf{g}}\left(\alpha_{g}\right)$, with $\mathbf{A}=\left[(0,1)^{T},(-1,0)^{T}\right]$, we can directly write:

$$
\frac{\partial \gamma_{g}}{\partial \alpha_{g}}=-s_{g} \sum_{i \in \mathscr{N}} \sum_{j \in \mathscr{N}} \mathbf{q}_{\mathbf{i j}}^{\mathbf{T}}\left(\mathbf{A} \mathbf{R}_{\mathbf{g}}\left(\alpha_{g}\right) \mathbf{c}_{\mathbf{i j}}\right)
$$


Let us define $\mathbf{c}_{\mathbf{i j}}^{\perp}=\mathbf{A} \mathbf{c}_{\mathbf{i j}}$. As $\mathbf{q}_{\mathbf{i j}}^{\mathbf{T}}\left(\mathbf{A} \mathbf{R}_{\mathbf{g}}\left(\alpha_{g}\right) \mathbf{c}_{\mathbf{i j}}\right)=-\sin \left(\alpha_{g}\right) \mathbf{q}_{\mathbf{i j}}^{\mathbf{T}} \mathbf{c}_{\mathbf{i j}}+\cos \left(\alpha_{g}\right) \mathbf{q}_{\mathbf{i j}}^{\mathbf{T}} \mathbf{c}_{\mathbf{i j}}^{\perp}$, we can make (6) equal to zero and solve for $\alpha_{g}$ to obtain the optimal angle. By doing so, one finds two candidate optimal angles $\alpha_{o c}$ that differ by $\pi$ rad. and satisfy the following expression:

$$
\alpha_{o c}=\arctan \frac{\sum_{i \in \mathscr{N}} \sum_{j \in \mathscr{N}} \mathbf{q}_{\mathbf{i j}}^{\mathbf{T}} \mathbf{c}_{\mathbf{i j}}^{\perp}}{\sum_{i \in \mathscr{N}} \sum_{j \in \mathscr{N}} \mathbf{q}_{\mathbf{i j}}^{\mathbf{T}} \mathbf{c}_{\mathbf{i j}}} .
$$

Let us denote:

$$
P=\sum_{i \in \mathscr{N}} \sum_{j \in \mathscr{N}} \mathbf{q}_{\mathbf{i j}}^{\mathbf{T}} \mathbf{c}_{\mathbf{i j}}, P^{\perp}=\sum_{i \in \mathscr{N}} \sum_{j \in \mathscr{N}} \mathbf{q}_{\mathbf{i j}}^{\mathbf{T}} \mathbf{c}_{\mathbf{i j}}^{\perp}
$$

It is straightforward to see that, out of the two candidate solutions of (7), the one that makes the second order derivative of $\gamma_{g}$ positive, i.e., the angle that minimises the function, is:

$$
\alpha_{o}=\operatorname{atan} 2\left(P^{\perp}, P\right) .
$$

The atan 2 function is the inverse tangent of the ratio of its two arguments, with the result placed in the quadrant that corresponds with the signs of the arguments. Observe that $\mathbf{R}_{\mathbf{0}}\left(\alpha_{o}\right)$ is independent from the scale. That is, the optimal rotation has the same yalue for any positive value of the scale $s_{g}$ in (4). Note that the $2 \pi$ discontinuity of atan 2 is not relevant as what the controller uses is not the angle $\alpha_{o}$ but its associated rotation matrix, which remains continuous in terms of $P^{\perp}$ and $P$. The case atan2 $(0,0)$ will be discussed below.

We find next the optimal scale. Differentiating (4) with respect to the scale parameter yields:

$$
\frac{\partial \gamma_{g}}{\partial s_{g}}=\sum_{i \in \mathscr{N}} \sum_{j \in \mathscr{N}} s_{g}|| \mathbf{c}_{\mathbf{i j}}||^{2}-\mathbf{q}_{\mathbf{i j}}^{\mathbf{T}} \mathbf{R}_{\mathbf{g}}\left(\alpha_{g}\right) \mathbf{c}_{\mathbf{i j}}
$$

Notice that we can already set $\mathbf{R}_{\mathbf{g}}\left(\alpha_{g}\right)=\mathbf{R}_{\mathbf{0}}\left(\alpha_{o}\right)$, as this rotation is the optimal one for any $s_{g}$, as explained above. Thus, making (10) equal to zero, one can easily find that the optimal scale is:

$$
s_{o}=\left(\sum_{i \in \mathscr{N}} \sum_{j \in \mathscr{N}} \mathbf{q}_{\mathbf{i j}}^{\mathbf{T}} \mathbf{R}_{\mathbf{o}}\left(\alpha_{o}\right) \mathbf{c}_{\mathbf{i j}}\right) / c_{s},
$$

where we have defined the strictly positive constant $c_{s}=\sum_{i \in \mathscr{N}} \sum_{j \in \mathscr{N}}\left\|\mathbf{c}_{\mathbf{i j}}\right\|^{2}$. Noticing that we can express $\sum_{i \in \mathscr{N}} \sum_{j \in \mathscr{N}} \mathbf{q}_{\mathbf{i j j}}^{\mathbf{T}} \mathbf{R}_{\mathbf{o}}\left(\alpha_{o}\right) \mathbf{c}_{\mathbf{i j}}=P^{\perp} \sin \left(\alpha_{o}\right)+P \cos \left(\alpha_{o}\right)$, we directly have:

$$
s_{o}=\left(P^{\perp} \sin \left(\alpha_{o}\right)+P \cos \left(\alpha_{o}\right)\right) / c_{s} .
$$

From the definition of $\alpha_{o}$ in (9), it can be readily seen that we can express: $P^{\perp}=K \sin \left(\alpha_{o}\right)$ and $P=K \cos \left(\alpha_{o}\right)$ for a certain scalar $K \geq 0$. Hence, it is direct from (12) that $s_{o}=K / c_{s}$. This means that the optimal scale is always positive and can only become zero (which occurs when $K=0$ ) in exactly the same configurations that make $\alpha_{o}$ degenerate. Indeed, $s_{o}=0 \Leftrightarrow$ $\left(P=0\right.$ and $\left.P^{\perp}=0\right)$ will be used to show that degeneracies do not occur with the proposed control methodology. Let us also state these alternative expressions for future use:

$$
P=s_{o} c_{s} \cos \left(\alpha_{o}\right), P^{\perp}=s_{o} c_{s} \sin \left(\alpha_{o}\right), P^{2}+{P^{\perp^{2}}}^{2}=s_{o}{ }^{2} c_{s}{ }^{2} .
$$


Considering the above, we can now define the following overall cost function:

$$
\gamma=\frac{1}{2} \sum_{i \in \mathscr{N}} \sum_{j \in \mathscr{N}}\left\|\mathbf{q}_{\mathbf{i j}}-s_{o} \mathbf{R}_{\mathbf{o}}\left(\alpha_{o}\right) \mathbf{c}_{\mathbf{i j}}\right\|^{2}
$$

where $\mathbf{R}_{\mathbf{0}}\left(\alpha_{o}\right)$ is computed via (9) and $s_{o}$ is obtained from (11). Then, we propose a gradientbased controller-described in the next section- defined from the cost function $\gamma$. Observe that the target is included in the function, and that if $\gamma=0$, the agents are forming the desired pattern, with the target in its centroid. We define an alternative cost function $\gamma_{-}$that only considers the interagent vectors (i.e., the target is not included):

$$
\gamma_{-}=\frac{1}{2} \sum_{i \in \mathscr{N}_{-}} \sum_{j \in \mathscr{N}_{-}}\left\|\mathbf{q}_{\mathbf{i j}}-s_{o} \mathbf{R}_{\mathbf{o}}\left(\alpha_{o}\right) \mathbf{c}_{\mathbf{i j}}\right\|^{2}
$$

and thus we have (observe that $\mathbf{q}_{\mathbf{i j}}=-\mathbf{q}_{\mathbf{j i}}, \mathbf{c}_{\mathbf{i j}}=-\mathbf{c}_{\mathbf{j i}}$ ):

$$
\gamma=\gamma_{-}+\sum_{i \in \mathscr{N}_{-}}\left\|\mathbf{q}_{\mathbf{i N}}-s_{o} \mathbf{R}_{\mathbf{o}}\left(\alpha_{o}\right) \mathbf{c}_{\mathbf{i N}}\right\|^{2} .
$$

Clearly, if $\gamma_{-}=0$ the agents are forming their desired optimal pattern, regardless of the position, $\mathbf{q}_{\mathbf{N}}$, of the target.

\section{Control laws}

We propose two different control laws for the agents under the following general formulation:

$$
\mathbf{u}_{\mathbf{i}}=\dot{\mathbf{q}}_{\mathbf{i}}=K_{c}\left(\mathbf{q} \mathbf{N i}-s \mathbf{R}(\alpha) \mathbf{c}_{\mathbf{N i}}\right), \quad i \in \mathscr{N}_{-},
$$

where $K_{c}$ is a positive control gain, $s>0$ is a scalar and $\mathbf{R}(\alpha) \in S O(2)$ is a rotation matrix. For later use, we express the relative agent dynamics with this control framework:

$$
\dot{\mathbf{q}}_{\mathbf{i j}}=\dot{\mathbf{q}}_{\mathbf{i}}-\dot{\mathbf{q}}_{\mathbf{j}}=-K_{c}\left(\mathbf{q}_{\mathbf{i j}}-s \mathbf{R}(\alpha) \mathbf{c}_{\mathbf{i j}}\right), \quad \forall i, j \in \mathscr{N}_{-} .
$$

The relative agent-target vectors evolve as follows:

$$
\dot{\mathbf{q}}_{\mathbf{i N}}=-K_{c}\left(\mathbf{q}_{\mathbf{i N}}-s \mathbf{R}(\alpha) \mathbf{c}_{\mathbf{i N}}\right)-\mathbf{v}_{\mathbf{t}}, \quad \forall i \in \mathscr{N}_{-} .
$$

We propose the following control laws, which are based on the expression (17) and use the optimal values $s_{o}(11)$ and $\alpha_{o}(9)$, computed at each time instant, as feedback parameters. Controller 1: This controller uses (17) with the optimal scale and rotation angle, i.e., $s=s_{o}$, $\alpha=\alpha_{0}$.

Controller 2: To achieve target circumnavigation, we select the control variables as follows: $s=s_{o} / \cos \left(\Delta_{\alpha}\right), \alpha=\alpha_{o}+\Delta_{\alpha}, \Delta_{\alpha}$ being a constant angle satisfying $0<\left|\Delta_{\alpha}\right|<\pi / 2$.

Remark 1. The individual agent control law (17) does not follow the negative gradient of the cost function (14). This is so because it also takes into account the objective of keeping the target in the group's centroid, and the fact that the target's free motion does not contribute to achieving the formation. The relative vector dynamics (18), (19) reveal, still, that the agents are indeed collectively minimising (14). 


\section{Controller analysis}

We provide next a number of results that characterise the behavior of the proposed approach. We will sometimes omit the angles of the rotation matrices, for notational simplicity.

\subsection{Behavior of the multiagent formation}

Lemma 5.1. The optimal scale $s_{o}$ remains constant with Controllers 1 and 2, i.e., $\dot{s}=\dot{s}_{o}=0$.

Proof. We compute the time derivative of $P(8)$ :

$$
\dot{P}=\sum_{i \in \mathscr{N}} \sum_{j \in \mathscr{N}}\left(\frac{\partial P}{\partial \mathbf{q}_{\mathrm{ij}}}\right)^{T} \dot{\mathbf{q}}_{\mathrm{ij}}
$$

Given that $\partial P / \partial \mathbf{q}_{\mathbf{i j}}=\mathbf{c}_{\mathbf{i j}}$ and inserting (18) and (19), we get:

$$
\begin{aligned}
\dot{P} & =\sum_{i \in N_{-}} \sum_{j \in N_{-}} \mathbf{c}_{\mathbf{i j}}^{\mathbf{T}}\left(-K_{c}\left(\mathbf{q}_{\mathbf{i j}}-s \mathbf{R} \mathbf{c}_{\mathbf{i j}}\right)\right)+2 \sum_{i \in \mathscr{N}_{-}} \mathbf{c}_{\mathbf{i N}}^{\mathbf{T}}\left(-K_{c}\left(\mathbf{q}_{\mathbf{i N}}-s \mathbf{R} \mathbf{c}_{\mathbf{i N}}\right)-\mathbf{v}_{\mathbf{t}}\right) \\
& =-K_{c} \sum_{i \in \mathscr{N}} \sum_{j \in \mathscr{N}} \mathbf{c}_{\mathbf{i j}}^{\mathbf{T}}\left(\mathbf{q}_{\mathbf{i j}}-s \mathbf{R} \mathbf{c}_{\mathbf{i j}}\right)-2 \mathbf{v}_{\mathbf{t}}^{\mathbf{T}} \sum_{i \in \mathscr{N}_{-}} \mathbf{c}_{\mathbf{i N}} .
\end{aligned}
$$

As said earlier, the desired position of the target is in the centroid of the desired pattern, i.e., $\sum_{i \in \mathscr{N}_{-}} \mathbf{c}_{\mathbf{i N}}=\sum_{i \in \mathscr{N}_{-}} \mathbf{c}_{\mathbf{i N}}^{\perp}=\mathbf{0}$. Therefore:

$$
\dot{P}=-K_{c} \sum_{i \in \mathscr{N}} \sum_{j \in \mathscr{N}} \mathbf{c}_{\mathbf{i j}}^{\mathbf{T}}\left(\mathbf{q}_{\mathbf{i j}}-s \mathbf{R} \mathbf{c}_{\mathbf{i j}}\right)=-K_{c}\left(\sum_{i \in \mathscr{K}} \sum_{j \in \mathscr{N}} \mathbf{c}_{\mathbf{i j}}^{\mathbf{T}} \mathbf{q}_{\mathbf{i j}}-s \sum_{i \in \mathscr{N}} \sum_{j \in \mathscr{N}} \mathbf{c}_{\mathbf{i j}}^{\mathbf{T}} \mathbf{R} \mathbf{c}_{\mathbf{i j}}\right) .
$$

Clearly, an analogous expression holds for $\dot{P}^{\perp}$.

$$
\dot{P}^{\perp}=-K_{c}\left(\sum_{i \in \mathscr{N}} \sum_{j \in \mathscr{N}} \mathbf{c}_{\mathbf{i j}}^{\perp} \mathbf{T} \mathbf{q}_{\mathbf{i j}}-s \sum_{i \in \mathscr{N}} \sum_{j \in \mathscr{N}} \mathbf{c}_{\mathbf{i j}}^{\perp} \mathbf{T} \mathbf{R}_{\mathbf{i j}}\right) .
$$

Now, substituting earlier definitions (13) and noticing that:

$$
\mathbf{c}_{\mathbf{i j}}^{\mathbf{T}}\left(\mathbf{R} \mathbf{c}_{\mathbf{i j}}\right)=\cos (\alpha)\left\|\mathbf{c}_{\mathbf{i j}}\right\|^{2}, \quad \mathbf{c}_{\mathbf{i j}}^{\perp \mathbf{T}}\left(\mathbf{R c}_{\mathbf{i j}}\right)=\sin (\alpha)\left\|\mathbf{c}_{\mathbf{i j}}\right\|^{2},
$$

(22) and (23) can be directly written as follows:

$$
\begin{aligned}
& \dot{P}=-K_{c}\left(P-\cos (\alpha) s c_{s}\right)=-K_{c} c_{s}\left(s_{o} \cos \left(\alpha_{o}\right)-s \cos (\alpha)\right) \\
& \dot{P}^{\perp}=-K_{c}\left(P^{\perp}-\sin (\alpha) s c_{s}\right)=-K_{c} c_{s}\left(s_{o} \sin \left(\alpha_{o}\right)-s \sin (\alpha)\right) .
\end{aligned}
$$

Let us now address the computation of $\dot{s}_{o}$. Consider (12), which expresses $s_{o}$ as a function of $\alpha_{o}, P^{\perp}$ and $P$. Computing $\partial s_{o} / \partial \alpha_{o}$ and then inserting the expressions (13), this partial derivative is directly seen to be zero. We can thus write:

$$
\dot{s}_{o}=\frac{\partial s_{o}}{\partial P^{\perp}} \dot{P}^{\perp}+\frac{\partial s_{o}}{\partial P} \dot{P}=\left(\sin \left(\alpha_{o}\right) \dot{P}^{\perp}+\cos \left(\alpha_{o}\right) \dot{P}\right) / c_{s} .
$$


Substituting now (25) in (26), we have:

$$
\begin{aligned}
\dot{s}_{o} & =-K_{c}\left(s_{o} \sin ^{2}\left(\alpha_{o}\right)-s \sin (\alpha) \sin \left(\alpha_{o}\right)+s_{o} \cos ^{2}\left(\alpha_{o}\right)-s \cos (\alpha) \cos \left(\alpha_{o}\right)\right) \\
& =-K_{c}\left(s_{o}-s \cos \left(\alpha-\alpha_{o}\right)\right) .
\end{aligned}
$$

Clearly, this expression is zero both for Controller 1 and Controller 2, and thus $s_{o}$ is constant. For Controller 1, moreover, $P$ and $P^{\perp}$ remain constant (see (25)).

Remark 2. As $s_{o}$ is constant for both controllers, degeneracies of $\alpha_{o}$ (9) cannot occur if $\alpha_{o}$ is initially non-degenerate (Section 3). Thus, $s_{o}>0$ and the control laws are always well defined. A constant scale is interesting in practice because it increases safety (in terms of, for instance, protection against collisions) and, as the distances between elements are steady) and upper bounded, the sensing/communications are more stable and robust.

Lemma 5.2. With Controller 1, the optimal rotation angle $\alpha=\alpha_{o}$ remains constant. With Controller 2 , the optimal angle varies with a constant speed $\dot{\alpha}=\dot{\alpha}_{0}=K_{c} \tan \left(\Delta_{\alpha}\right)$.

Proof. Controller 1: Given that $\dot{P}=0$ and $\dot{P}^{\perp}=0$ (Lemma 5.1), $\dot{\alpha}_{o}=0$ (see (9)). Controller 2: As $\alpha_{o}(9)$ is always well defined and differentiable -see Remark 2-, we can express its time derivative as:

$$
\dot{\alpha}_{o}=\frac{d\left(P^{\perp} / P\right) / d t}{1+\left(P^{\perp} / P\right)^{2}}=\frac{P \dot{P}^{\perp}-P^{\perp} \dot{P}}{P^{2}+P^{\perp^{2}}} .
$$

Direct substitution of (13), (25) and straightforward manipulations lead to:

$$
\dot{\alpha}_{o}=\frac{-K_{c}}{s_{o}} s\left(\sin \left(\alpha_{o}\right) \cos (\alpha)-\cos \left(\alpha_{o}\right) \sin (\alpha)\right)=K_{c} \tan \left(\Delta_{\alpha}\right),
$$

which is the stated result.

Remark 3. A constant $\alpha_{o}$ is useful as it reduces the actuation effort for the agents. In particular, with Controller 1, the target will remain -assuming a small error in its tracking- in the same direction relative to each agent, thus an agent can more easily maintain it in its fieldof-view. The target circumnavigation allowed by Controller 2 is interesting as it provides a more complete target representation, avoiding perceptual occlusions. It also makes it harder for the target to escape the enclosing, if this is desired. With our method the gyrating speed is controllable by design and constant, which is advantageous as it results in a smooth motion.

Theorem 5.3. The agents converge exponentially to the optimal desired formation with Controllers 1 and 2. With Controller 1, the agents converge to a pattern that remains fixed along time. With Controller 2 , they converge to a pattern that gyrates with constant angular velocity equal to $K_{c} \tan \left(\Delta_{\alpha}\right)$.

Proof. Consider for the analysis the dynamics in (18).

Controller 1. Since both $s=s_{o}$ and $\alpha=\alpha_{o}$ are constant (Lemmas 5.1 and 5.2), we can directly conclude the exponential convergence of every interagent vector $\mathbf{q}_{\mathbf{i j}}$ to its constant desired vector $s_{o} \mathbf{R}_{\mathbf{o}}\left(\alpha_{o}\right) \mathbf{c}_{\mathbf{i j}}$.

Controller 2. We have:

$$
\dot{\mathbf{q}}_{\mathbf{i j}}=-K_{c}\left(\mathbf{q}_{\mathbf{i j}}-\left(s_{o} / \cos \left(\Delta_{\alpha}\right)\right) \mathbf{R}(\alpha) \mathbf{c}_{\mathbf{i j}}\right), \forall i, j \in \mathscr{N}_{-} .
$$


where $s_{o}$ is constant and $\alpha=\alpha(t=0)+K_{c} \tan \left(\Delta_{\alpha}\right) t=\alpha_{o}(t=0)+\Delta_{\alpha}+K_{c} \tan \left(\Delta_{\alpha}\right) t$ (Lemmas 5.1 and 5.2). Assume for simplicity and without loss of generality that $\alpha_{o}(t=0)=0$. Solving the differential equation (30) yields:

$$
\mathbf{q}_{\mathbf{i j}}(t)=\left(\mathbf{q}_{\mathbf{i j}}(0)-s_{o} \mathbf{c}_{\mathbf{i j}}\right) e^{-K_{c} t}+s_{o} \mathbf{R}\left(K_{c} \tan \left(\Delta_{\alpha}\right) t\right) \mathbf{c}_{\mathbf{i j}}
$$

Thus, $\mathbf{q}_{\mathrm{ij}}$ converges exponentially to a circular orbit of radius $s_{o}|| \mathbf{c}_{\mathbf{i j}} \|$, along which it moves at fixed speed. As (31) holds for every vector $\mathbf{q}_{\mathbf{i j}}, \forall i, j \in \mathscr{N}_{-}$, the full formation with constant optimal scale is achieved exponentially, while it rotates with constant speed $K_{c} \tan \left(\Delta_{\alpha}\right)$.

\subsection{Target tracking performance}

After dealing with the interagent behaviors, we now address how the control system performs in regard to tracking the target, i.e., we characterise its ability to maintain the target enclosed and as close as possible to the centroid of the multiagent team.

Theorem 5.4. For Controllers 1 and 2 , the centroid $\mathbf{p}_{\mathbf{q}}$ of the multiagent group tracks the target at all times with a velocity $\dot{\mathbf{p}}_{\mathbf{q}}(t)=K_{c}\left(\mathbf{q}_{\mathbf{N}}(t)-\mathbf{p}_{\mathbf{q}}(t)\right)$. For Controller 1 , moreover, the velocity of every one of the agents converges exponentially to this tracking velocity.

Proof. For both Controller 1 and 2, the dynamics of the centroid can be computed using (17) as follows:

$$
\dot{\mathbf{p}}_{\mathbf{q}}=\frac{1}{N-1} \sum_{i \in \mathscr{N}_{-}} \dot{\mathbf{q}}_{\mathbf{i}}=\frac{K_{c}}{N-1}\left(\sum_{i \in \mathcal{N}_{-}} \mathbf{q}_{\mathbf{N i}}-s \mathbf{R} \sum_{i \in \mathscr{N}_{-}} \mathbf{c}_{\mathbf{N i}}\right) .
$$

Given that $\sum_{i \in \mathscr{N}_{-}} \mathbf{c}_{\mathbf{N i}}=\mathbf{0}$ due to the target's desired position being at the centroid of the prescribed pattern, we have:

$$
\dot{\mathbf{p}}_{\mathbf{q}}=\frac{K_{c}}{N-1} \sum_{i \in \mathscr{N}} \mathbf{q}_{\mathbf{N}}=\frac{K_{c}}{N-1}\left((N-1) \mathbf{q}_{\mathbf{N}}-\sum_{i \in \mathscr{N}_{-}} \mathbf{q}_{\mathbf{i}}\right)=K_{c}\left(\mathbf{q}_{\mathbf{N}}-\mathbf{p}_{\mathbf{q}}\right) .
$$

Thus, the centroid purely tracks the target, and its evolution is completely decoupled from the formation geometry.

Controller 1. When the agents are forming the prescribed pattern, the scaling and rotation of this pattern are obviously equal to $s_{o}$ and $\mathbf{R}_{\mathbf{0}}$. Each position vector from the current centroid to a given agent is then equal to the optimally scaled and rotated vector from the centroid (occupied by the target) to the agent in the desired pattern. Thus, for Controller 1:

$$
\mathbf{q}_{\mathbf{i}}-\mathbf{p}_{\mathbf{q}}=s_{o} \mathbf{R}_{\mathbf{o}} \mathbf{c}_{\mathbf{i N}}, \forall i \in \mathscr{N}_{-},
$$

a condition that will be reached with exponential convergence (see Theorem 5.3). Therefore, it is direct from (17) that the difference between the agent velocities will converge to zero, i.e., all the agents will have the following velocity:

$$
\dot{\mathbf{q}}_{\mathbf{i}}=K_{c}\left(\mathbf{q}_{\mathbf{N i}}-s_{o} \mathbf{R}_{\mathbf{0}} \mathbf{c}_{\mathbf{N i}}\right)=K_{c}\left(\left(\mathbf{q}_{\mathbf{N}}-\mathbf{q}_{\mathbf{i}}\right)+\left(\mathbf{q}_{\mathbf{i}}-\mathbf{p}_{\mathbf{q}}\right)\right)=K_{c}\left(\mathbf{q}_{\mathbf{N}}-\mathbf{p}_{\mathbf{q}}\right), \forall i \in \mathscr{N}_{-},
$$

as claimed in the statement of this result.

It is then clear that for both controllers, the tracking behavior is equivalent to that of a virtual agent placed in the team's centroid which continuously tracks the target's position (i.e., 

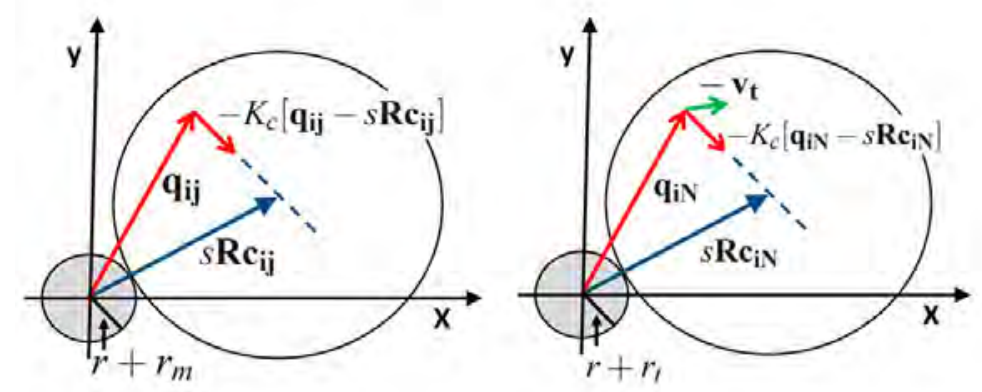

Figure 1. Illustration of collision avoidance. $s=s_{o}, \mathbf{R}=\mathbf{R}_{\mathbf{0}}$.

tries to capture it). The target's speed $\left\|\mathbf{v}_{\mathbf{t}}\right\|$, the gain $K_{c}$ and the maximum speed attainable by the agents determine how closely this tracking is achieved. Note also that the angular speed of the pattern, $K_{c} \tan \left(\Delta_{\alpha}\right)$, is decoupled from the formation achievement and target tracking performances (dependent on $K_{c}$ ), because $\Delta_{\alpha}$ can be selected freely.

\subsection{Collision avoidance}

We provide next conditions under which collisions will not occur for the proposed target enclosing methodology. We model every agent as a disk of physical radius $r$, and the target as a disk of radius $r_{t}$. We denote $r_{m}=\max \left(r, r_{t}\right)$, and assume that $s_{o}\left\|\mathbf{c}_{\mathbf{i j}}\right\|>r+r_{m} \forall i, j \in \mathscr{N}$.

Theorem 5.5. For Controllers 1 and 2, if the initial configuration is such that $\| \mathbf{q}_{\mathbf{i j}}-$ $s_{o} \mathbf{R}_{\mathbf{0}} \mathbf{c}_{\mathbf{i j}}\left\|<s_{o}\right\| \mathbf{c}_{\mathbf{i j}} \|-\left(r+r_{m}\right) \forall i, j \in \mathscr{N}$, there are no interagent collisions. If, furthermore, the speed of the target is bounded as $\left\|\mathbf{v}_{\mathbf{t}}\right\|<K_{c}\left(s_{0} \min _{i}\left(\left\|\mathbf{c}_{\mathbf{i N}}\right\|\right)-\left(r+r_{t}\right)\right)$, there are no collisions between any agent and the target with Controller 1.

Proof. The arguments are illustrated in Fig. 1 .

Interagent collisions. Agents $i$ and $j$ collide if $\mathbf{q}_{\mathbf{i j}}$ enters the collision circle defined by $\| \mathbf{q}_{\mathbf{i j j}}||<\left(r+r_{m}\right)$. $\mathbf{q}_{\mathbf{i j}}$ is initially in the safety circle $\left\|\mathbf{q}_{\mathbf{i j}}-s_{o} \mathbf{R}_{\mathbf{o}} \mathbf{c}_{\mathbf{i j}}\right\|<s_{o}|| \mathbf{c}_{\mathbf{i j}} \|-\left(r+r_{m}\right)$. Observe that due to the exponential convergence (Theorem 5.3), it is direct to see that each vector $\mathbf{q}_{\mathbf{i j}}$ (18) gets monotonically closer to its desired reference $s_{o} \mathbf{R}_{\mathbf{0}} \mathbf{c}_{\mathbf{i j}}$, either constant (Controller 1) or gyrating (Controller 2). This readily implies that it cannot leave the safety circle.

Agent-target collisions. Agent $i$ and the target collide if $\mathbf{q}_{\mathbf{i N}}$ enters the circle defined by $\left\|\mathbf{q}_{\mathbf{i N}}\right\|<\left(r+r_{t}\right) \cdot \mathbf{q}_{\mathbf{i N}}$ is initially in the safety circle $\left\|\mathbf{q}_{\mathbf{i N}}-s_{o} \mathbf{R}_{\mathbf{0}} \mathbf{c}_{\mathbf{i N}}\right\|<s_{o}\left\|\mathbf{c}_{\mathbf{i N}}\right\|-\left(r+r_{t}\right)$ (recall $r_{t} \leq r_{m}$ ). The controller pulls it directly towards the centre of the circle (the constant reference $\left.s_{o} \mathbf{R}_{\mathbf{0}} \mathbf{c}_{\mathbf{i N}}\right)$; let us denote this formation vector as: $\mathbf{v}_{\mathbf{i N}}=-K_{c}\left(\mathbf{q}_{\mathbf{i N}}-s \mathbf{R}(\alpha) \mathbf{c}_{\mathbf{i N}}\right)$, so that (19) is $\dot{\mathbf{q}}_{\mathbf{i N}}=\mathbf{v}_{\mathbf{i N}}-\mathbf{v}_{\mathbf{t}} \forall i \in \mathscr{N}_{-}$. Thus, clearly, if $\left\|\mathbf{v}_{\mathbf{t}}\right\|<\left\|\mathbf{v}_{\mathbf{i N}}\right\|$ then $\dot{\mathbf{q}}_{\mathbf{i N}}^{T} \mathbf{v}_{\mathbf{i N}}>0$, which implies $\mathbf{q}_{\mathrm{iN}}$ will remain in the circle. In particular, it suffices to ensure this condition when $\mathbf{q}_{\mathbf{i N}}$ is on the edge of the circle. In that case, $\left\|\mathbf{v}_{\mathbf{i N}}\right\|=K_{c}\left(s_{o}|| \mathbf{c}_{\mathbf{i N}} \|-\left(r+r_{t}\right)\right)$, so the condition is satisfied for all $i$ if $\left\|\mathbf{v}_{\mathbf{t}}\right\|<K_{c}\left(s_{o} \min _{i}\left(\left\|\mathbf{c}_{\mathbf{i N}}\right\|\right)-\left(r+r_{t}\right)\right)$, as stated. Observe that collisions among more than two agents are ruled out under the conditions of the Theorem, since these conditions are formulated for every pair of agents $i, j$. In addition, note that the controller has not been modified in our collision avoidance study. Therefore, all the stability results obtained in Section 5 remain valid.

The intuition behind this analysis is that there are no collisions if the team is sufficiently close to the desired formation initially. The stated conditions depend on the scale $s_{o}$, which will be typically high enough when the agents are initially sufficiently separated, and close to the formation. Agent-target collisions with Controller 2 behave similarly to Controller 1, but 
they are more involved to analyse due to the desired formation vectors rotating over time.

We will finish our analysis of the controller with the observations that follow.

Remark 4. In gradient-based undirected formation control, systematic errors either in the measurements or in the formation's prescription lead to persistent group motions, which may be rectilinear (Dimarogonas and Kyriakopoulos (2008)) or circular (García de Marina, Cao, and Jayawardhana (2015); Mou, Belabbas, Morse, Sun, and Anderson (2016)). For our controller, such errors might lead to circular group motions around the target -a behavior similar to that of Controller 2-. Also, drifts of the formation scale due to these inconsistencies can be typically compensated by the agents via appropriate adjustment of the value of $s$.

Remark 5. Switching between Controllers 1 and 2 enables interesting flexible behaviors. For instance, the agents can start gyrating around the pattern when they need to perceive occluded parts of it, or when the target slows down and thus it becomes safer and more comfortable for the target and less energy-consuming for the agents to circumnavigate it. They can also choose to selectively rotate the multiagent pattern so as to guarantee safe navigation in the presence of external obstacles. Importantly, as the scale is constant and equal for both controllers, such back-and-forth switches will be seamless. Also, there are ways to ensure in practice that the scale is lower bounded with the proposed controllers, e.g., agents with knowledge of distances may use it to steer the team's optimal scale (one agent suffices for this). The assignment of agents to places in the formation can be exploited as well, as it also influences the scale value.

\section{Method implementation}

We discuss next the information needed to compute the proposed controller. A key fact is that the method can be implemented by each agent using its local measurements, in a completely decentralised manner. Indeed, in order to calculate its control input, it is sufficient for an agent $k$ to measure the relative positions of the other agents and the target with respect to itself (i.e., $\mathbf{q}_{\mathbf{j k}}, \forall j \in \mathscr{N}$ ). Note that from these measurements, $k$ can directly compute all the vectors it needs (i.e., all $\mathbf{q}_{\mathbf{j i}}$ when $j \neq k, i \neq k$ ). Therefore, communications are clearly not needed.

For the purposes of the technical analysis, in the paper we express all the variables of the controller in a global reference frame. However, a crucial property is that the agents do not need to have access to this common global reference: indeed, the control can be computed if each agent uses a local and arbitrarily oriented coordinate frame, as illustrated next. First, as discussed in the previous paragraph, all position measurements $\left(\mathbf{q}_{\mathbf{j i}}\right)$ used are relative, so no common coordinate origin for the different agents is needed. Furthermore, the specific orientation of each agent's reference frame is irrelevant. To see this, let us define $\mathbf{P}_{\mathbf{k}} \in S O(2)$ as the rotation matrix between the global frame and the local frame in which agent $k$ operates. We denote with a superscript $L k$ the variables expressed in $k^{\prime} s$ local frame and we have, then, that $\mathbf{q}_{\mathbf{i j}}^{\mathbf{L}} \mathbf{k}=\mathbf{P}_{\mathbf{k}} \mathbf{q}_{\mathbf{i j}} \forall i, j \in \mathscr{N}$. We next look at (4), and assume given fixed positions $\mathbf{q}_{\mathbf{i}} \forall i \in \mathscr{N}$. We can write (we do not notate the angles of the rotations, for increased simplicity):

$$
\begin{aligned}
\gamma_{g}^{L k}\left(s_{g}^{L k}, \mathbf{R}_{\mathbf{g}}^{\mathbf{L k}}\right) & =\frac{1}{2} \sum_{i \in \mathscr{N}} \sum_{j \in \mathscr{N}}\left\|\mathbf{q}_{\mathbf{i j}}^{\mathbf{L k}}-s_{g}^{L k} \mathbf{R}_{\mathbf{g}}^{\mathbf{L k}} \mathbf{c}_{\mathbf{i j}}\right\|^{2} \\
& =\frac{1}{2} \sum_{i \in \mathscr{N}} \sum_{j \in \mathscr{N}}\left\|\mathbf{q}_{\mathbf{i j}}-s_{g}^{L k} \mathbf{P}_{\mathbf{k}}^{-\mathbf{1}} \mathbf{R}_{\mathbf{g}}^{\mathbf{L k}} \mathbf{c}_{\mathbf{i j}}\right\|^{2}=\gamma_{g}\left(s_{g}^{L k}, \mathbf{P}_{\mathbf{k}}^{-\mathbf{1}} \mathbf{R}_{\mathbf{g}}^{\mathbf{L k}}\right) .
\end{aligned}
$$

As explained in Section 3, the optimal rotation is independent from the scale (that is, from 
$s_{g}^{L k}$ in (36)). Hence, it is direct to see that the unique rotations in the two frames involved that solve the optimisation problem (5) must be such that $\mathbf{R}_{\mathbf{0}}=\mathbf{P}_{\mathbf{k}}^{-\mathbf{1}} \mathbf{R}_{\mathbf{0}}^{\mathbf{L k}}$, i.e., $\mathbf{R}_{\mathbf{o}}^{\mathbf{L k}}=\mathbf{P}_{\mathbf{k}} \mathbf{R}_{\mathbf{0}}$. Let us now examine the expression for the optimal scale (11) when computed in the local frame:

$$
s_{o}^{L k}=\left(\sum_{i \in \mathscr{N}} \sum_{j \in \mathscr{N}} \mathbf{q}_{\mathbf{i j}}^{\mathbf{L k}}{ }^{\mathbf{T}} \mathbf{R}_{\mathbf{o}}^{\mathbf{L k}} \mathbf{c}_{\mathbf{i j}}\right) / c_{s}=\left(\sum_{i \in \mathscr{N}} \sum_{j \in \mathscr{N}}\left(\mathbf{P}_{\mathbf{k}} \mathbf{q}_{\mathbf{i j}}\right)^{\mathbf{T}}\left(\mathbf{P}_{\mathbf{k}} \mathbf{R}_{\mathbf{o}} \mathbf{c}_{\mathbf{i j}}\right)\right) / c_{s} .
$$

As both vectors in each of the scalar products are rotated by the same matrix $\mathbf{P}_{\mathbf{k}}$, it is direct, by comparing (37) and (11), to see that $s_{o}^{L k}=s_{o}$. Observe that for the parameters of Controller 2 (Section 4), one easily sees that $s^{L k}=s$ and (denoting as $\mathbf{R}_{\Delta_{\alpha}}$ the rotation by an angle $\Delta_{\alpha}$ ) it also holds that $\mathbf{R}^{\mathbf{L k}}=\mathbf{R}_{\Delta_{\alpha}} \mathbf{R}_{\mathbf{o}}^{\mathbf{L k}}=\mathbf{R}_{\Delta_{\alpha}} \mathbf{P}_{\mathbf{k}} \mathbf{R}_{\mathbf{o}}=\mathbf{P}_{\mathbf{k}} \mathbf{R}_{\Delta_{\alpha}} \mathbf{R}_{\mathbf{o}}=\mathbf{P}_{\mathbf{k}} \mathbf{R}$. We can then write down the common expression for both controllers (17) when computed in $k^{\prime} s$ local frame:

$$
\mathbf{u}_{\mathbf{k}}^{\mathbf{L k}}=K_{c}\left(\mathbf{q}_{\mathbf{N k}}^{\mathbf{L k}}-s^{L k} \mathbf{R}^{\mathbf{L k}} \mathbf{c}_{\mathbf{N k}}\right)=K_{c}\left(\mathbf{P}_{\mathbf{k}} \mathbf{q}_{\mathbf{N k}}-s \mathbf{P}_{\mathbf{k}} \mathbf{R} \mathbf{c}_{\mathbf{N k}}\right)=\mathbf{P}_{\mathbf{k}} \mathbf{u}_{\mathbf{k}}
$$

Hence, one directly concludes that the exact same motion is obtained when the control is computed in each of the two frames.

We also note that in a possible case where $k$ can only measure some (i.e. not all) of its neighbors' relative positions, then it can obtain the remaining measurements via communications with other agents. By using exchanged relative measurements, $k$ can reconstruct the vectors it can not directly measure, and also estimate the differences in orientation between local frames, so that the information received from other agents is integrated consistently. Let us remark that the computational cost of the proposed approach is low, because the optimal parameters -angle (9) and scale (11)- can be computed analytically.

\subsection{Implementation without distance measurements}

The control law (17) can be computed even if the agents do not possess information of distances. To see this, consider an agent that can measure (e.g., using angular sensors) the relative positions of the other agents and the target up to a given time-varying unknown scale. That is, agent $k$ knows $r_{k} \mathbf{q}_{\mathrm{ij}}$ for all $i, j \in \mathcal{N}$, with $r_{k}>0$. Then, looking at (9), notice that $k$ can compute an optimal angle with its no-distance data (we notate this with a superindex $n d k$ ):

$$
\begin{aligned}
\alpha_{o}^{n d k} & =\operatorname{atan} 2\left(\sum_{i \in \mathscr{N}} \sum_{j \in \mathscr{N}} r_{k} \mathbf{q}_{\mathbf{i j}}^{\mathbf{T}} \mathbf{c}_{\mathbf{i j}}^{\perp}, \sum_{i \in \mathscr{N}} \sum_{j \in \mathscr{N}} r_{k} \mathbf{q}_{\mathbf{i j}}^{\mathbf{T}} \mathbf{c}_{\mathbf{i j}}\right) \\
& =\operatorname{atan} 2\left(r_{k} P^{\perp}, r_{k} P\right)=\operatorname{atan} 2\left(P^{\perp}, P\right)=\alpha_{o}
\end{aligned}
$$

i.e., it computes the same optimal angle as in the case where distance information is available. In addition, the agent can also compute an optimal scale (see (11)):

$$
s_{o}^{n d k}=\left(\sum_{i \in \mathscr{N}} \sum_{j \in \mathscr{N}} r_{k} \mathbf{q}_{\mathbf{i j}}^{\mathbf{T}} \mathbf{R}\left(\alpha_{o}\right) \mathbf{c}_{\mathbf{i j}}\right) / c_{s}=r_{k} s_{o},
$$

i.e., the result it obtains is weighted by its own unknown scale. Then, if $k$ computes its control law (either for Controller 1 or 2) following (17), it will have:

$$
\begin{aligned}
\mathbf{u}_{\mathbf{k}} & =K_{c}\left(r_{k} \mathbf{q}_{\mathbf{N k}}-s^{n d k} \mathbf{R}^{\mathbf{n d k}}\left(\alpha^{n d k}\right) \mathbf{c}_{\mathbf{N k}}\right)=K_{c}\left(r_{k} \mathbf{q}_{\mathbf{N k}}-r_{k} s \mathbf{R}(\alpha) \mathbf{c}_{\mathbf{N k}}\right) \\
& =r_{k}\left(K_{c}\left(\mathbf{q}_{\mathbf{N k}}-s \mathbf{R}(\alpha) \mathbf{c}_{\mathbf{N k}}\right)\right) .
\end{aligned}
$$


Thus, the control law is the same as when the distances are known, except for a weighting of the motion control action by a scale factor $r_{k}$. We can define a common scale reference for all agents in a direct way by, e.g., making all of them impose the constraint $\left\|q_{i j}\right\|=1$ for a given nonzero measurement $(i, j)$, and scale the magnitude of all their position measurements accordingly. Then, all agents will share the same scale factor, $r_{k}=r_{g}$ for all $k$, and an effective control gain $r_{g} K_{c}$. Clearly, the expressions for the evolutions of the optimal scale and angle in Lemmas 5.1 and 5.2 are independent from the value and time variation of the control gain, as long as this value is always common to all agents. Thus, the desirable types of behaviors of the controller analysed in Section 5 will be maintained. Note also that if an agent employs measurements received from other agents, it is possible to correct the relative scale inconsistencies existing in its data without needing absolute distance measurements.

Let us finally describe a possible practical implementation setup. Each robot can carry an omnidirectional vision sensor (as in, e.g., the work (Das, Fierro, Kumar, Ostrowski, Spletzer, and Taylor (2002)). Using the size in the image of the viewed agents or the size of visual markers, it is possible for a robot to directly measure the relative position vectors from itself to the other robots and the target. In a general case, these vectors are expressed in the robot's image frame, and their length is equal to the actual metric distance multiplied by an unknown scale factor. These measurements are sufficient to implement the controller.

\section{Simulation examples}

We evaluate next the proposed method in simulation. The target moved following sinusoidal patterns in all tests. Fig. 2 illustrates the first three examples we present. In the first of them, Controller 1 was used by a team of eight agents with a square-shaped prescribed geometry. It can be seen that the desired enclosing behavior materialised and, as theoretically expected, a constant optimal scale (equal to 0.97 , with respect to the prescribed formation), and pattern rotation angle ( $0.78 \mathrm{rad}$, in an arbitrary fixed reference frame) were obtained. Also, the cost function $\gamma_{-}$(15) vanished exponentially, while $\gamma$ did not, due to the persistent motion of the target. We illustrate another example of Controller 1 with a six-agent team and a triangleshaped desired geometry. To test the robustness of the proposed approach, we added Gaussian noise to each relative position measurement used by the agents. In addition, we defined the control gain as equal for all agents, but time-varying throughout the execution, to model the case where distance measurements are not available (see Section 6.1). The effects of noise were apparent, but the team exhibited a satisfactory behavior. In particular, neither the angles nor the scales of the pattern computed by the different agents drifted during the execution.

We also report on an execution of Controller 2 with a scalene triangular desired pattern. The plots show that the three agents converged towards this pattern. The scale $s$ kept a value equal to 2.22. As the agents circumnavigated the target, the distance between their centroid and the target remained small thanks to the tracking properties of the controller.

Finally, we provide a comparison (illustrated in Fig. 3) of our method with the target encirclement approach presented in Franchi et al. (2016). We used a four-robot team and a circular desired pattern. We implemented our method using Controller 2, which produces a target circumnavigation behavior. To provide a balanced comparison, we selected the parameters in the two methods such that in both cases the agents reached an equivalent final configuration and gyrating speed, with identical initial positions and very similar initial velocities, and identical motion of the target. Both methods can be seen to provide suitable performance. The approach in Franchi et al. (2016) has more robustness and scalability because it uses distributed coordination. Also, it has strong collision avoidance guarantees and is directly applicable in 
3D space. The method we propose is arguably more flexible in the team geometries that can be chosen, and achieves the desired enclosing configuration of the system more quickly. In addition, our method relaxes some information requirements, because neither distance measurements nor communications are needed. We compare the cost functions $\left(\gamma, \gamma_{-}\right)$as they encapsulate the control objective for the two approaches. It must be noted, however, that the controller in Franchi et al. (2016) is not designed to optimise these cost functions.

\section{Conclusion}

This paper introduced a novel method to perform the task of enclosing a moving target in a two dimensional environment with a team of mobile agents. The proposed approach is interesting because it requires few resources from the agents and exploits the information available in an optimal manner towards solving the problem at hand. An analysis that reveals the uniform variation of the multiagent pattern's rotation and the constancy of its scale serves us to show that the team exhibits steady behaviors, even in the absence of common references shared by the agents. The use of global information is reasonable for tasks where few robots are used and for which stable and predictable behaviors are of particular relevance. Still, it would be clearly interesting to improve the robustness to failure of the proposed method. One direction to follow in this respect can be to study the case of temporary agent faults. Another idea can be to use only partial information, searching for a balance between the amount of information used and the steadiness and stability of behavior. Aside from investigating these issues, other appealing topics for future work include non-holonomic motion models, and the definition of optimal desired formation geometries based on task-related criteria.

\section{References}

Anderson, B. D. O., Yu, C., Fidan, B., Hendrickx, J. M., 2008. Rigid graph control architectures for autonomous formations. IEEE Control Systems 28 (6), 48-63.

Aranda, M., López-Nicolás, G., Saguiés, C., Zavlanos, M. M., 2014. Three-dimensional multirobot formation control for target enclosing. In: IEEE/RSJ Int. Conf. on Intell. Robots and Systems. pp. $357-362$.

Aranda, M., López-Nicolás, G., Saguiés, C., Zavlanos, M. M., 2015. Coordinate-free formation stabilization based on relative position measurements. Automatica 57, 11-20.

Bishop, A. N., Fidan, B., Anderson, B. D. O., Doanay, K., Pathirana, P. N., 2010. Optimality analysis of sensor-target localization geometries. Automatica 46 (3), $479-492$.

Cao, Y., Stuart, D., Ren, W., Meng, Z., 2011. Distributed Containment Control for Multiple Autonomous Vehicles With Double-Integrator Dynamics: Algorithms and Experiments. IEEE Transactions on Control Systems Technology 19 (4), 929-938.

Cao, Y., Zhang, L., Li, C., Chen, M. Z. Q., 2017. Observer-Based Consensus Tracking of Nonlinear Agents in Hybrid Varying Directed Topology. IEEE Transactions on Cybernetics 47 (8), 2212-2222.

Das, A. K., Fierro, R., Kumar, V., Ostrowski, J. P., Spletzer, J., Taylor, C. J., 2002. A vision-based formation control framework. IEEE Transactions on Robotics and Automation 18 (5), 813-825.

Dimarogonas, D. V., Kyriakopoulos, K. J., 2008. A connection between formation infeasibility and velocity alignment in kinematic multi-agent systems. Automatica 44 (10), 2648-2654.

Eren, T., 2012. Formation shape control based on bearing rigidity. International Journal of Control 85 (9), 1361-1379.

Franchi, A., Stegagno, P., Oriolo, G., 2016. Decentralized multi-robot encirclement of a 3D target with guaranteed collision avoidance. Autonomous Robots 40 (2), 245-265.

Franchi, A., Stegagno, P., Rocco, M. D., Oriolo, G., 2010. Distributed target localization and encir- 

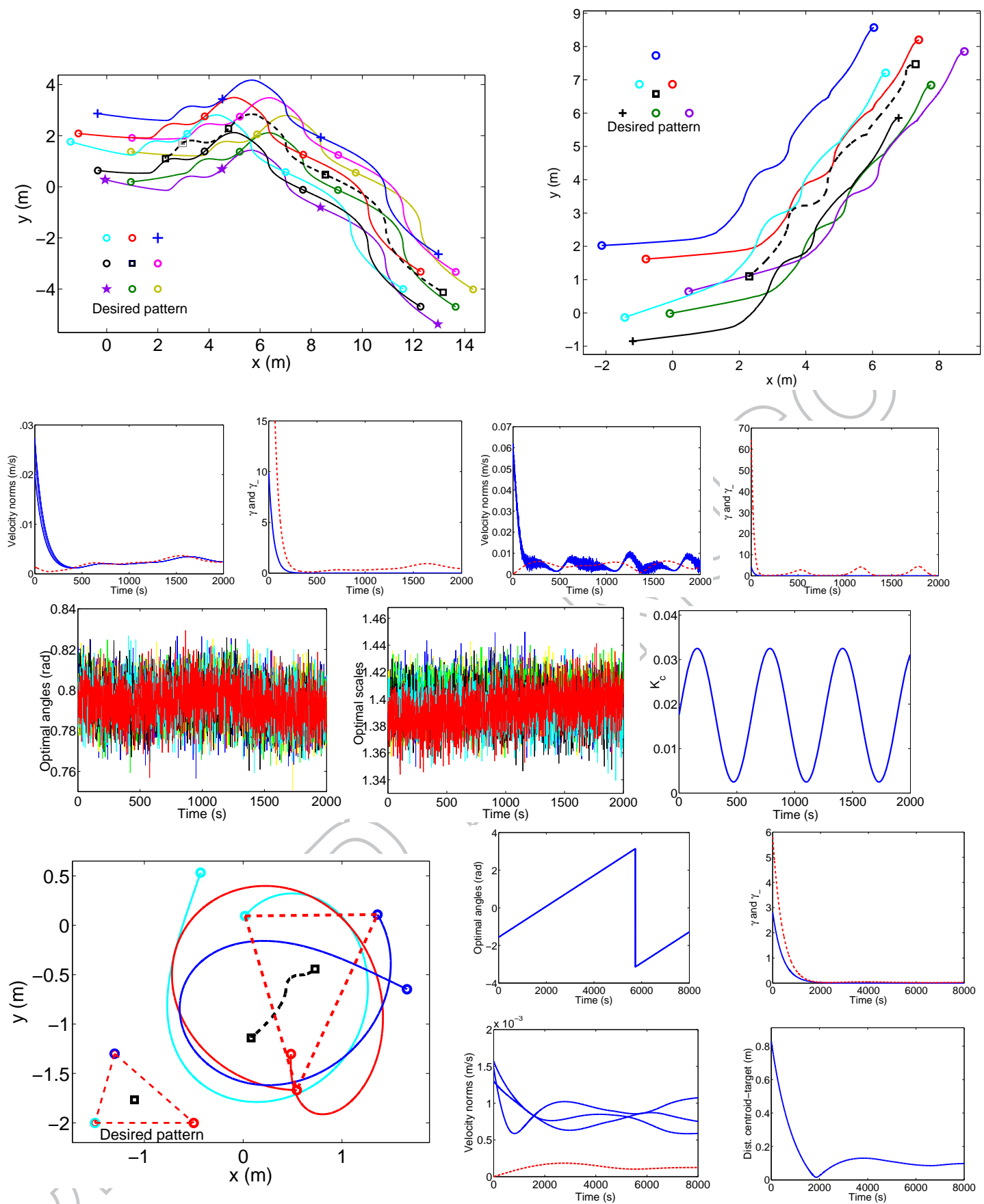

Figure 2. Simulations. Top row: agents' and target's paths for example 1 (left) and example 2 (right). Agents are circles different markers and colors are also used to ease interpretation-, target is a square and its path is shown in dashed line. Initial, final and some intermediate positions of the elements are marked. Second row, left to right: velocity norms for agents -solid line- and target -dashed line-, and cost functions (always $\gamma \geq \gamma_{-}$) for example 1 ; velocity norms, and cost functions, for example 2 with Gaussian noise. Third row: optimal angles (left) and scales (centre), and control gain (right) for example 2. Bottom-left: agents' and target's paths for example 3. Final agent positions joined by dashed lines. Bottom-right panel, top: optimal angles (left) and cost functions (right) for example 3. Bottom: velocity norms and centroid-target distance for example 3 . 

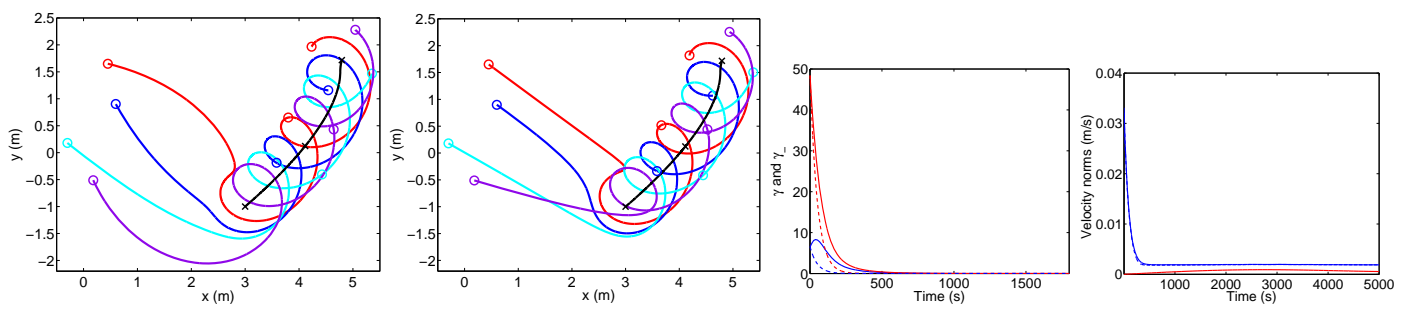

Figure 3. Results for comparative simulation example. Left to right: agent and target paths for the method in Franchi et al. (2016) and for the approach proposed in this paper (initial, final and intermediate positions of agents -circles-and target -crossare marked); Cost functions (our method in dashed line), where the higher-valued curves are $\gamma$; average velocities of the agents (our method in dashed line) and velocity of the target (lower-valued curve).

clement with a multi-robot system. 7th IFAC Symp. Intellig. Autonomous Vehicles, IFAC Proceed. Volumes 43 (16), 151-156.

García de Marina, H., Cao, M., Jayawardhana, B., 2015. Controlling rigid formations of mobile agents under inconsistent measurements. IEEE Transactions on Robotics 31 (1), 31-39.

Garcia de Marina, H., Jayawardhana, B., Cao, M., 2016. Distributed rotational and translational maneuvering of rigid formations and their applications. IEEE Transactions on Robotics 32 (3), 684-697.

Gower, J. C., Dijksterhuis, G. B., 2004. Procrustes problems. Oxford University Press.

Guerra-Filho, G., 2005. Optical motion capture: Theory and implementation. Journal of Theoretical and Applied Informatics 12 (2), 61-89.

Guo, J., Yan, G., Lin, Z., 2010. Cooperative control synthesis for moving-target-enclosing with changing topologies. In: IEEE International Conference on Robotics and Automation. pp. 1468-1473.

Hausman, K., Müller, J., Hariharan, A., Ayanian, N., Sukhatme, G. S., 2015. Cooperative multi-robot control for target tracking with onboard sensing. The International Journal of Robotics Research 34 (13), 1660-1677.

Ji, M., Ferrari-Trecate, G., Egerstedt, M., Buffa, A., 2008. Containment control in mobile networks. IEEE Transactions on Automatic Control 53 (8), 1972-1975.

Khan, A., Rinner, B., Cavallaro, A., 2017. Cooperative robots to observe moving targets: Review. IEEE Transactions on Cybernetics PP (99), 1-12.

Krick, L., Broucke, M. E., Francis, B. A., 2009. Stabilisation of infinitesimally rigid formations of multi-robot networks. International Journal of Control 82 (3), 423-439.

Kruse, T., Pandey, A. K., Alami, R., Kirsch, A., 2013. Human-aware robot navigation: A survey. Robotics and Autonomous Systems 61 (12), 1726 - 1743.

Lin, Z., Wang, L., Han, Z., Fu, M., 2014. Distributed formation control of multi-agent systems using complex Laplacian. IEEE Trans. Autom. Control 59 (7), 1765-1777.

Macdonald, E. A., 2011. Multi-robot assignment and formation control. Masters thesis. Georgia Institute of Technology.

Mallik, G. R., Daingade, S., Sinha, A., 2016. Scalable multi-agent formation with bearing only measurement: Consensus based approach. European Journal of Control 27, 28-35.

Marasco, A., Givigi, S., Rabbath, C.-A., 2012. Model predictive control for the dynamic encirclement of a target. In: American Control Conference. pp. 2004-2009.

Martínez, S., Bullo, F., 2006. Optimal sensor placement and motion coordination for target tracking. Automatica 42 (4), $661-668$.

Mas, I., Li, S., Acain, J., Kitts, C., 2009. Entrapment/escorting and patrolling missions in multi-robot cluster space control. In: IEEE/RSJ Int. Conf. on Intelligent Robots and Systems. pp. 5855-5861.

Montijano, E., Priolo, A., Gasparri, A., Sagüés, C., 2013. Distributed entrapment for multi-robot systems with uncertainties. In: IEEE Conf. Decis. Contr. pp. 5403-5408.

Mou, S., Belabbas, M. A., Morse, A. S., Sun, Z., Anderson, B. D. O., 2016. Undirected rigid formations are problematic. IEEE Trans. Automatic Control 61 (10), 2821-2836.

Oh, K.-K., Park, M.-C., Ahn, H.-S., 2015. A survey of multi-agent formation control. Automatica 53, 424-440. 
Poiesi, F., Cavallaro, A., 2015. Distributed vision-based flying cameras to film a moving target. In: IEEE/RSJ Intern. Conf. on Intell. Rob. and Systems. pp. 2453-2459.

Ren, W., 2010. Consensus Tracking Under Directed Interaction Topologies: Algorithms and Experiments. IEEE Transactions on Control Systems Technology 18 (1), 230-237.

Robin, C., Lacroix, S., 2016. Multi-robot target detection and tracking: taxonomy and survey. Autonomous Robots 40 (4), 729-760.

Shames, I., Fidan, B., Anderson, B. D. O., 2011. Close target reconnaissance with guaranteed collision avoidance. Int. Journ. Robust and Nonlinear Control 21 (16), 1823-1840.

Shi, Y. J., Li, R., Teo, K. L., 2015. Cooperative enclosing control for multiple moving targets by a group of agents. International Journal of Control 88 (1), 80-89.

Swartling, J. O., Shames, I., Johansson, K. H., Dimarogonas, D. V., 2014 Collective circumnavigation. Unmanned Systems 2 (3), 219-229.

Tian, Y.-P., Wang, Q., 2013. Global stabilization of rigid formations in the plane. Automatica 49 (5), $1436-1441$.

Wang, Y.-W., Liu, X.-K., Xiao, J.-W., Lin, X., 2017. Output formation-containment of coupled heterogeneous linear systems under intermittent communication. Journal of the Franklin Institute 354 (1), 392-414.

Wang, Y.-W., Liu, X.-K., Xiao, J.-W., Shen, Y., 2018. Output formation-containment of interacted heterogeneous linear systems by distributed hybrid active control. Automatica 93, 26-32.

Ze-Su, C., Jie, Z., Jian, C., 2012. Formation control and obstacle avoidance for multiple robots subject to wheel-slip. Int. Journ. Advanced Robotic Syst. 9 (5), 188-202.

Zelazo, D., Giordano, P. R., Franchi, A., 2015. Bearing-only formation control using an SE(2) rigidity theory. In: IEEE Conference on Decision and Control. pp. 6121-6126.

Zhao, S., Chen, B. M., Lee, T. H., 2013. Optimal sensor placement for target localisation and tracking in 2D and 3D. International Journal of Control 86 (10), 1687-1704.

Zheng, R., Liu, Y., Sun, D., 2015. Enclosing a target by nonholonomic mobile robots with bearing-only measurements. Automatica 53, 400-407.

Zhou, K., Roumeliotis, S. I., 2011. Multirobot active target tracking with combinations of relative observations. IEEE Transactions on Robotics 27 (4), 678-695. 